MATEC Web of Conferences 16, 10006 (2014)

DOI: $10.1051 /$ matecconf/ 20141610006

(C) Owned by the authors, published by EDP Sciences, 2014

\title{
Damage identification in beams by a response surface based technique
}

\author{
S. Teidj, A. Driouach, A. Khamlichi
}

Communications Systems and Detection Laboratory; Abdelmalek Essaadi University, Tetouan 93002, Morocco

\begin{abstract}
In this work, identification of damage in uniform homogeneous metallic beams was considered through the propagation of non dispersive elastic torsional waves. The proposed damage detection procedure consisted of the following sequence. Giving a localized torque excitation, having the form of a short half-sine pulse, the first step was calculating the transient solution of the resulting torsional wave. This torque could be generated in practice by means of asymmetric laser irradiation of the beam surface. Then, a localized defect assumed to be characterized by an abrupt reduction of beam section area with a given height and extent was placed at a known location of the beam. Next, the response in terms of transverse section rotation rate was obtained for a point situated afterwards the defect, where the sensor was positioned. This last could utilize in practice the concept of laser vibrometry. A parametric study has been conducted after that by using a full factorial design of experiments table and numerical simulations based on a finite difference characteristic scheme. This has enabled the derivation of a response surface model that was shown to represent adequately the response of the system in terms of the following factors: defect extent and severity. The final step was performing the inverse problem solution in order to identify the defect characteristics by using measurement.
\end{abstract}




\section{Introduction}

Defects in beams can be generated by various fracture mechanisms that range from anodyne surface flaws, to severe internal cracks and finally to complete transverse breakages. In the particular case of rails, Cannon et al. [1] have given a classification of common defects.

In carrying out maintenance of beams, periodic inspections based on non destructive evaluation (NDE) are scheduled. The aim is to detect defects before they lead to sudden wrecking of members. Small cracks can in some circumstances grow very fast without giving the possibility to detect them on time. The ability of detecting small defects before they become dangerous can enhance dramatically safety issues, especially in transportation systems.

With a rhythm of inspections that is slow, the risk to experience total breakage of beam members during in service operational time cannot be discarded with acceptable confidence. Thus, intensifying inspections is definitely required in order to limit such unexpected breakage events that could have tragic consequences such as train derailment. Reducing inspection charge is then a key challenge in order to get better maintenance practices in this field. Quick NDE techniques are also desirable in order to perform the various diagnosis tasks in almost real time and with minimum lost caused by immobilisation of the equipment.

Considering metallic beams suffering a damage of transverse crack type, traditional techniques work generally by monitoring the impedance along the system. However, this technique cannot work consistently for a beam section which is not almost completely broken, as the impedance may not show significant measurable change. So, alternate methods have been proposed. Eddy current based technology is one of them. However, the limited penetration depth of eddy currents restricts detection to only the surface and close subsurface defects of the examined system. Moreover, the eddy current sensor is sensitive to any variations in the electromagnetic properties and also to the distance separating the receiver coil from the target which can vary because of the beam surface roughness. Thus, singular points are also detected. To correct the obtained predictions by eddy current based technique, a Bayesian based method which uses two sources of information in order to distinguish between actual broken sections and ordinary singular points was proposed [2].

Other inspection methods based on ultrasonic wave propagation have been proposed [3]. To detect internal flaws, very short ultrasonic pulse-waves with main frequencies ranging from $0.1-50 \mathrm{MHz}$ are launched into the material. This non destructive technique suffers nonetheless some shortcomings as it is not always capable of detecting deep cracks in rails. The presence of superficial cracks can cause earlier reflections which impede ultrasonic waves to penetrate sufficiently into the core of the structure [4]. False predictions are current and statistics have revealed that almost $75 \%$ of predictions made by ultrasonic base technique were false.

New inspection methods have been introduced recently to enhance detection of internal flaws. Some of them use the concept of elastic wave propagation [5]. This technique consists of generating high frequency mechanical stress waves in the ultrasonic range. These take the general form of guided waves which have the ability to travel along a large distance with little loss in energy. Damage detection relies then on the fact that the transient pattern will change if a defect is present on the pathway between the excitation and the measurement points. Rose et al. [5] have investigated the use of elastic guided wave propagation in rails to detect transverse cracks. The method consists of generating elastic waves from the train itself and measuring the response by a set of transducers positioned at pertinent locations. This allowed correct determination of rail breaks, but the requirement for further installations on the track limits of course its applicability for supervision of real railways networks.

New ideas have emerged recently about monitoring generation and detection of elastic guided waves by mounting contactless devices that can achieve both excitation and measurement. Such systems can deliver information about section failure even in intensive operating condition. Considering rails systems, Coccia et al. [6] have presented a modelling of the interaction resulting from a source laser excitation with some typical defects affecting the rail head. The free-vibration and the forced solutions under this kind of excitation of the rail surface were calculated up to a frequency of $500 \mathrm{kHz}$. The analysis allowed for the identification of certain wave modes that are more suitable for specific types of rail head defects. Recently, a prototype based on this technology to generate ultrasonic waves in the rail, and an array of air-coupled sensors to detect the waves was built and tested [7]. The results have shown good sensitivity to transverse-type defects, yielding significant improvement of reliability of detection. The inspection speed was higher than those of the conventional rail defect detection methods. Precision was found to be better as the tests proved to separate satisfactorily good welds from defective ones.

To detect the waves, the previous authors used aircoupled sensors. These can however limit the performance of the system as the ratio of noise to signal could be rather high. Considering propagation of torsional waves in rails, a laser torsional vibrometer (LTV) has been found to be suitable for measurement of beam section rotation rate $[8,9]$. This sensor, which relies on the principle of laser Doppler velocimetry, offers real reward over the conventional techniques as it is contactless and yet more accurate. Coupling a source laser with a LTV to assemble an embedded compact inspection device system that can be mounted underneath a railway vehicle is liable to provide a fast method for rail inspection.

In the present work, focus is on local defects affecting straight uniform beams which take the general form of transverse flaws for which a loss of rigidity is experienced at the section where they are located. The characteristics of a defect of this kind can be roughly described by two parameters: the extent of the defect and its severity (loss of height). Pulsed guided waves that develop from a laser spot radiation impacting the beam 
head system and which excite predominantly the torsional modes of the structure are considered. The frequency content of these waves is taken high in order to maximize spatial resolution. Simulation of torsional wave propagation is performed in order to get the response in terms of rotational velocity at any given section of the beam where a LTV sensor is raised. This is achieved in the following by using a finite difference scheme base on the method of characteristics [10].

In the presence of damage, partial transmission and reflection will occur at the section where it is located. This causes modification of the transmitted rotation and rotation velocity peaks. These peaks will carry then essential information about the defect that can be readily correlated to the defect characteristics. To find this correlation, results are generated according to a full factorial design of experiment table will enable derivation of a response surface. This explicit form can be used to perform inversion of the problem in order to get the defect characteristics from measurement. As these characteristics of the modelled defect include two parameters, two independent equations will be required to achieve this inversion. These equations are provided by the peaks of the rotation and the rotation rate. They are nonlinear, their solution can be calculated by using a standard Gauss-Siedel method.

\section{Propagation of torsional elastic waves in a damaged beam}

Propagation of elastic waves in beam which is likely to suffer a localized transverse crack, figure 1 , can be readily performed by using a finite difference scheme based on the method of characteristics [10]. Special attention is given to the mesh to be used and to the time step in order to satisfy the stability condition. For defects that are small, enough discretisation points should be placed at the section of the defect. Pulse duration is fixed enough small to enable a resolution that is comparable of that of the defect extent.

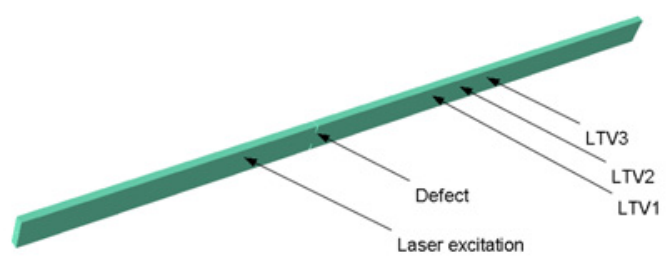

Fig. 1. A beam showing a localized transverse crack and positions of laser excitation point and laser vibrometer.

The beam is assumed to be made from a linear homogeneous and isotropic elastic material. The excitation is chosen such that it generates mainly a system of torsional waves that propagate along the longitudinal direction. Such an excitation can be provided by a special laser device that illuminates asymmetrically the beam head $[6,11]$. To be effective in detecting a small size flaw having a characteristic length greater than $\delta$ the work excitation frequency should be at least $\delta / c_{T}$ where $c_{T}$ is the torsional wave velocity given by

$c_{T}=\sqrt{\frac{G \gamma}{\rho J_{p}}}$

where $G$ is the shear modulus, $\rho$ the material density, $\gamma$ the torsion constant and $J_{p}$ the polar moment of inertia.

It is assumed that the effect of laser excitation could be assimilated to that of a torque generated at the irradiated section of the beam. The torque is denoted in the following $C(t)$ where $t$ designates time. A sensor using laser vibrometry concept is positioned at another given section of the beam. In this section, the instantaneously rotational velocity $\dot{\theta}(t)$ can then be measured by using Doppler effect. This signal will be continuously recorded as the travelling torsional pulse crosses that section.

Written in terms of the velocity $\dot{\theta}(t)$, the equation of propagation for torsional waves in a beam writes

$G \gamma \frac{\partial^{2} \dot{\theta}}{\partial x^{2}}=\rho J_{p} \frac{\partial^{2} \dot{\theta}}{\partial t^{2}}$

where $x$ is the axial coordinate of the beam. This equation is subjected to boundary and initial conditions.

During simulation, the duration of time interval is chosen such that no extra signal comes from the exiting boundary conditions that could alter the resulting wave pattern at the measurement section.

In the presence of a discontinuity that can be assimilated to a sudden variation of the beam section, like that shown in figure 1, the torsional wave will be subjected to reflection/transmission phenomenon at that interface. Denoting respectively 1 and 2 the two adjacent parts of the beam separated by the discontinuity interface, the coefficients of reflection and transmission are given by the following equations

$$
\begin{aligned}
& r=\frac{\rho_{1} J_{p 1} c_{T 1}-\rho_{2} J_{p 2} c_{T 2}}{\rho_{1} J_{p 1} c_{T 1}+\rho_{2} J_{p 2} c_{T 2}} \\
& \tau=\frac{2 \rho_{1} J_{p 1} c_{T 2}}{\rho_{1} J_{p 1} c_{T 1}+\rho_{2} J_{p 2} c_{T 2}}
\end{aligned}
$$

From equation (4), one can notice that the transmitted wave will transmit information about the section variation, namely the defect extent (size) and depth (severity).

\section{Damage detection based on response surface modelling}

A simple mathematical relationship can be derived between the observed output in terms of rotation of rotation rate peak and the input independent variables that 
consist of defect characteristics: defect size (b) and defect severity $(\varepsilon)$. This mathematical relationship can be found by performing surface fitting. This encompasses the choice of an appropriate mathematical expression to fit the observed data values before using the fitted surface function to predict the output for the considered input variables within the considered ranges.

Different fitting methods can be employed to interpolate the input data in order to find the fitting surface model parameters. Each method has its own criteria for evaluating the fitting residual that is used for regression purposes.

The coefficient of determination denoted $R^{2}$ is a commonly used statistics criterion that serves to examine adequacy the fitting model. $R^{2}$ is equal to 1 minus the ratio of residual variability. When the variability of the residual values around the regression surface with regards to the overall variability is small, the predictions from the proposed regression equation are judged to be good. Otherwise, the proposed mathematical fitting curve is inadequate since it can not apprehend the nonlinearities that are inherent to the process.

Analysis of variance consists of calculations that provide information about levels of variability within a regression model which form the basis for tests of significance. The basic regression line concept, Data $=$ Fit + Residual, can be rewritten as follows

$y_{i}-\bar{y}=\left(\hat{y}_{i}-\bar{y}\right)+\left(y_{i}-\hat{y}_{i}\right)$

The term in the left half side is the total variation in the response $y$, the first term in the second half side is the variation as observed with regards to the proposed regression model, and the second term is the residual value. This should vanish if the model were exact. Squaring each of these terms and adding over all of the $N$ observations gives the equation

$\sum_{i=1}^{N}\left(y_{i}-\bar{y}\right)^{2}=\sum_{i=1}^{N}\left(\hat{y}_{i}-\bar{y}\right)^{2}+\sum_{i=1}^{N}\left(y_{i}-\hat{y}_{i}\right)^{2}$

This equation may also be written as $S S T=S S M+S S E$, where $S S$ is used to denote the sum of squares and $T$, $M$ and $E$ are notations that refer respectively to total, model and error.

The square of the sample correlation is equal to the ratio of the model sum of squares to the total sum of squares

$R^{2}=S S M / S S T=1-S S E / S S T$

Here a quadratic polynomial is proposed for regression of the obtained results in terms of the parameters $b$ and $\varepsilon$. A full factorial design of experiment table is used then in order to determine the six polynomial coefficients. The general form of this quadratic polynomial is given as follows

$\hat{y}=\alpha_{0}+\alpha_{1} b+\alpha_{2} \varepsilon+\alpha_{3} b \varepsilon+\alpha_{4} b^{2}+\alpha_{5} \varepsilon^{2}$
The full factorial table will provide 9 results and the coefficients $\alpha_{i}, i=0, \ldots, 6$ will be computed in the least square sense. Note that these coefficients don't depend on the laser excitation pulse location and the LTV location. Maintaining fixed the point of excitation and the LTV position; one can measure the rotation rate and integrate to obtain the rotation. These signals can be processed to get their peaks. A system of two nonlinear equations having the general form of equation (8) and which unknowns are the parameters $b$ and $\varepsilon$ can be obtained

$\left\{\begin{array}{l}\alpha_{0}+\alpha_{1} b+\alpha_{2} \varepsilon+\alpha_{3} b \varepsilon+\alpha_{4} b^{2}+\alpha_{5} \varepsilon^{2}=\dot{\theta}_{\text {max }} \\ \beta_{0}+\beta_{1} b+\beta_{2} \varepsilon+\beta_{3} b \varepsilon+\beta_{4} b^{2}+\beta_{5} \varepsilon^{2}=\theta_{\text {max }}\end{array}\right.$

Inversion of system of equations (9) can yield then the defect characteristics. For this purpose the two correlations used should be enough adequate with a high $R^{2}$ value each.

\section{Results and discussion}

A beam having the geometry depicted in figure 1 is considered. The beam of length $L=1 \mathrm{~m}$ has a uniform rectangular cross section of area $A=4 \times 10^{-4} \mathrm{~m}^{4}$. It has a constant mass per unit length $\mu=1.064 \mathrm{~kg} \cdot \mathrm{m}^{-1}$, Young's modulus $E=67.4 G P a$ and Poisson coefficient $v=0.3$. This gives a shear modulus $G=25.9 G P a$.

Denoting $w$ the width of the section and $h$ its height, the polar inertia and the torsion constant are respectively given by

$J_{p}=\frac{h w\left(h^{2}+w^{2}\right)}{12}$

$\gamma=\frac{16}{3} w h^{3}\left[1-\frac{192 h}{w \pi^{5}} \sum_{n=1,3, . .}^{\infty} \frac{1}{n^{5}} \tanh \left(\frac{n \pi w}{2 h}\right)\right]$

For $w<h$, equation (11) can be approximated by the following formula

$\gamma=h w^{3}\left[\frac{1}{3}-0.21 \frac{w}{h}\left(1-\frac{w^{4}}{12 h^{4}}\right)\right]$

Using equations (1), (10) and (12) the torsional wave speed writes

$$
c_{T}=c_{S} \sqrt{\frac{4-2.52 \frac{w}{h}\left(1-\frac{w^{4}}{12 h^{4}}\right)}{1+\left(\frac{h}{w}\right)^{2}}}
$$

where $c_{S}=\sqrt{G / \rho}$ is the velocity of shear waves. The shear velocity has here the value $c_{S}=3118 \mathrm{~m} \cdot \mathrm{s}^{-1}$.

Equation (13) shows that the torsional velocity of waves depends on the ratio $w / h$. In the presence of a defect for which the section height looses symmetrically 
a part of its total height denoted $\varepsilon$, which fixes then defect severity, the torsional wave velocity becomes

$c_{T}(\varepsilon)=c_{S} \sqrt{\frac{4-2.52 \frac{w}{(h-\varepsilon)}\left(1-\frac{w^{4}}{12(h-\varepsilon)^{4}}\right)}{1+\left(\frac{h-\varepsilon}{w}\right)^{2}}}$

This velocity caries then information about the defect present in that section.

The excitation torque is applied at the section located at the distance $0.3 m$ from the left extremity. The LTV is assumed to be positioned at the section having the abscissa $0.7071 \mathrm{~m}$ measured from the origin taken at the left extremity of the beam. The defect is chosen to have the following characteristics: $b \in\{0.5,1,1.5\} \mathrm{mm}$ and $\mathcal{E} \in\{0.005,0.010,0.015\} \mathrm{mm}$. Table 1 summarizes the chosen three levels of these two factors.

Table 1. Levels of the defect characteristics.

\begin{tabular}{|l|c|c|c|}
\hline & $\begin{array}{c}\text { Low } \\
\text { value }\end{array}$ & $\begin{array}{c}\text { Median } \\
\text { value }\end{array}$ & $\begin{array}{c}\text { High } \\
\text { value }\end{array}$ \\
\hline $\begin{array}{c}\text { Defect size; } \\
b \text { in }(\mathrm{mm})\end{array}$ & 0.5 & 1 & 1.5 \\
\hline $\begin{array}{c}\text { Defect severity; } \\
\varepsilon \text { in }(\mathrm{mm})\end{array}$ & 0.005 & 0.010 & 0.015 \\
\hline
\end{tabular}

Using equation (12) and the three values of defect height as given respectively by the last line of table 1 , one obtains respectively the following torsional wave speeds $c_{T 1}=1553 \mathrm{~m} \cdot \mathrm{s}^{-1}, \quad c_{T 2}=1755 \mathrm{~m} \cdot \mathrm{s}^{-1}, \quad c_{T 3}=2006 \mathrm{~m} \cdot \mathrm{s}^{-1}$. The intact beam torsion wave speed is $c_{T 0}=1390 \mathrm{~m} \cdot \mathrm{s}^{-1}$. One can notice that the wave speed increases with increasing defect height and that the changes are important.

The undamaged beam was modelled at first. Then the beam suffering from a localised flaw of magnitude $\varepsilon$ and extending over the length $b$ was modelled for each combination of these factors as obtained from table 1. A total number of nine combinations are possible.

The applied torque was chosen to have a half-sine profile which is generated by the following function

$$
C(t)=C_{\max } \sin ^{2}\left(\frac{\pi t}{T}\right)
$$

with $C_{\max }=10^{5} \mathrm{~N} . \mathrm{m}^{-1}$ and $T=1.0277 \times 10^{-6} \mathrm{~s}$. The total time interval for calculating the solution was fixed at $T_{c}=5 \times 10^{-4} \mathrm{~s}$.

A finite difference scheme based on the method of characteristics was developed by using Matlab. A uniform spatial discretisation was used with fixed step at $\Delta x=0.07143 \mathrm{~mm}$. The time step was determinate by the CFL conditions at fixed $\Delta t=5.139 \times 10^{-8} s$. The boundary conditions used correspond to fixed extremities, but these lasts don't intervene in the form of the considered signal because of the small chosen interval time for the calculation of the transient solution.

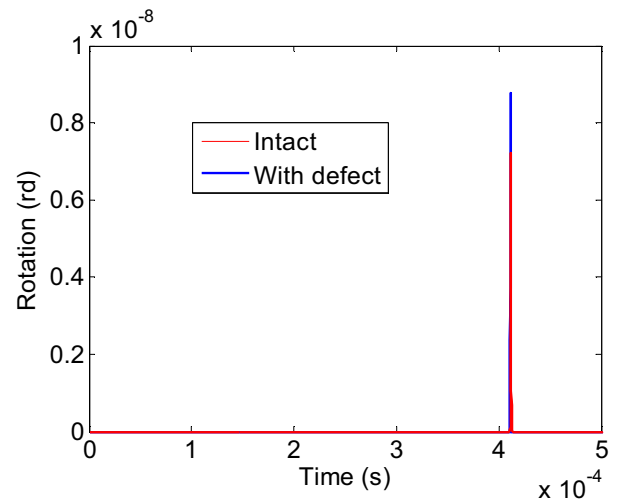

Fig. 2. Rotation about the $x$-axis for the intact beam and the defected beam with defect characteristics corresponding to $(b, \mathcal{E})=(1.5,0.015) \mathrm{mm}$.

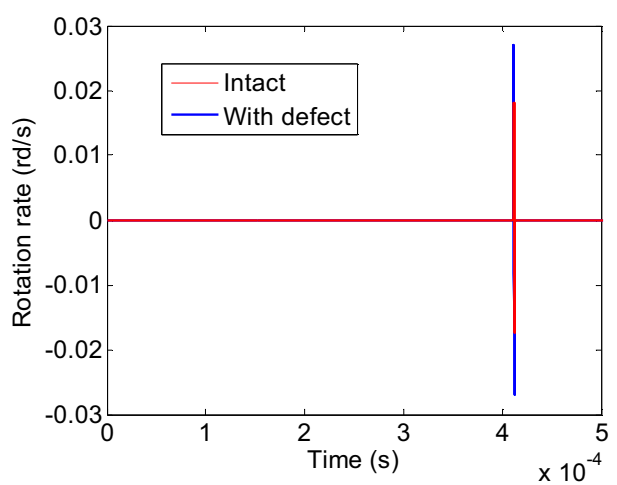

Fig. 3. Rotation rate about the $x$-axis for the intact beam and the defected beam with defect characteristics corresponding to $(b, \varepsilon)=(1.5,0.015) \mathrm{mm}$.

Table 2 gives the obtained peak of rotation velocity as function of the considered combination.

Table 3 gives the obtained rotation peak as function of the considered combination.

Analysis of variance associated to the interpolation of results by equation (9) has shown an excellent $R^{2}$ as the obtained values have exceeded $99.9 \%$. This justifies the use of a regression having the form of a quadratic polynomial. Even a linear interpolation can be used with an adequacy of $R^{2}=95 \%$.

Both the two factors were found to influence the outputs.

The quadratic polynomials giving respectively the rotation rate peak and the rotation peak as function of the factors are given in terms of $\bar{b}=b / \Delta x$ and $\varepsilon$ by

$$
\begin{aligned}
& \dot{\theta}_{\max }=2.5302 \times 10^{-2}-5.5524 \times 10^{-4} \bar{b}+0.13333 \varepsilon \\
& +2.1357 \times 10^{-2} \bar{b} \mathcal{\varepsilon}+3.0952 \times 10^{-6} \bar{b}^{2}+6.6667 \times 10^{-2} \varepsilon^{2} \\
& \theta_{\max } / \Delta t=0.16622-1.8809 \times 10^{-3} \bar{b} \\
& +0.6 \varepsilon+8.5714 \times 10^{-2} \bar{b} \varepsilon+3.4014 \times 10^{-6} \bar{b}^{2}-13.333 \varepsilon^{2}
\end{aligned}
$$

Table 2. Maximum rotation speed as function of the considered combination. 


\begin{tabular}{|c|c|c|c|}
\hline & $\bar{b}=b / \Delta x$ & $\varepsilon(\mathrm{mm})$ & $\begin{array}{c}\dot{\theta}_{\max } \times 10^{2} \\
\left(\mathrm{rd} . \mathrm{s}^{-1}\right)\end{array}$ \\
\hline 1 & 7 & 0.005 & 2.3 \\
\hline 2 & 7 & 0.01 & 2.44 \\
\hline 3 & 7 & 0.015 & 2.58 \\
\hline 4 & 14 & 0.005 & 2.02 \\
\hline 5 & 14 & 0.01 & 2.26 \\
\hline 6 & 14 & 0.015 & 2.46 \\
\hline 7 & 21 & 0.005 & 1.8 \\
\hline 8 & 21 & 0.01 & 2.07 \\
\hline 9 & 21 & 0.015 & 2.38 \\
\hline
\end{tabular}

Table 3. Maximum rotation as function of the considered combination.

\begin{tabular}{|c|c|c|c|}
\hline & $\bar{b}=b / \Delta x$ & $\varepsilon(\mathrm{mm})$ & $\begin{array}{c}\theta_{\max } \times 10^{9} \\
(\mathrm{rd})\end{array}$ \\
\hline 1 & 7 & 0.005 & 8.17 \\
\hline 2 & 7 & 0.01 & 8.41 \\
\hline 3 & 7 & 0.015 & 8.61 \\
\hline 4 & 14 & 0.005 & 7.66 \\
\hline 5 & 14 & 0.01 & 8.05 \\
\hline 6 & 14 & 0.015 & 8.46 \\
\hline 7 & 21 & 0.005 & 7.21 \\
\hline 8 & 21 & 0.01 & 7.76 \\
\hline 9 & 21 & 0.015 & 8.30 \\
\hline
\end{tabular}

Lets now consider the direct problem for which $(\bar{b}, \varepsilon)=(18,0.012)$. The calculated outputs in terms of arrival time are: $\dot{\theta}_{\max }=2.25 \times 10^{-2} r d . s^{-1}$ and $\theta_{\max } / \Delta t=0.157$.

Using equations (16) and (17) solving the nonlinear system by Gauss-Seidel scheme, one obtains the following values of parameters $(\bar{b}, \varepsilon)=(18.2,0.015)$ which is not far from the exact solution.

One can see that the input data was recovered with good accuracy, showing that the approach could be used to identify a transverse defect occurring on a beam by solution of an inverse problem.

\section{Conclusions}

A technique based on torsional elastic wave propagation and response surface meta-modelling was presented for damage identification in homogenous straight uniform beams. A finite difference scheme based on the method of characteristics was used to simulate the problem and to calculate the transient response under a pulse torque excitation, in terms of section rotation rate and rotation. This information as altered by the presence of a defect has enabled correlating the obtained rotation rate and rotation peaks with the factors describing the characteristics of the defect. The correlation used was a quadratic polynomial and has been found to achieve high $\mathrm{R}$-square value for both these outputs. Inverse problem solution was performed then by inverting the two nonlinear quadratic equations in order to identify the defect characteristics. The obtained results were encouraging. However sensitivity of the method should still be assessed as function of the variability that could affect the various intervening parameters.

\section{References}

1. D.F. Cannon, K.O. Edel, S.L. Grassie, K. Sawley, Fatigue and Fracture of Engineering Materials and Structures, 26 (2003)

2. L. Oukhellou, E. Côme, L. Bouillaut, P. Aknin. Transportation Research Part C, 16 (2008)

3. G. Zumpano, M. Meo, International Journal of Solids and Structures, 43 (2006)

4. J.J. Marais, K.C. Mistry, Fatigue and Fracture of Engineering Materials and Structures, 26 (2003)

5. J. Rose, M. Avioli, P. Mudgeand, R. Sanderson, Non Destructive Testing \& Evaluation International, 37, 2 (2004)

6. S. Coccia, I. Bartoli, A. Marzani, F. Lanza di Scalea, S. Salamone, M. Fateh, NDT\&E International, 44 (2011)

7. S. Coccia, R. Phillips, I. Bartoli, S. Salamone, P. Rizzo, F. Lanza di Scalea, On-line high-speed rail defect detection, Part II (DOT/FRA/ORD-12/02, Washington, 2012)

8. L. Xiang, S. Yang, C. Gan, Optics and Lasers in Engineering, 50 (2012)

9. S.J. Rothberg, M. Tirabassi, Mechanical Systems and Signal Processing, 26 (2012)

10. L. Kaishin, L. Bin. International Journal of Solids and Structures, 38, 48-49 (2001)

11. H. Yamawaki, T. Saito, Nondestructive Testing and Evaluation, 7, 1-6 (1992) 